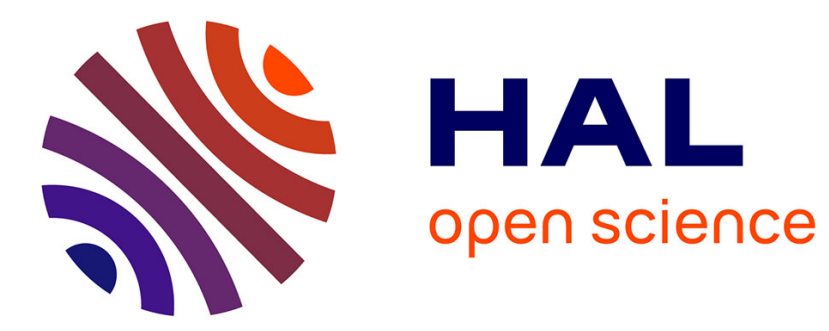

\title{
Spherical cap bubbles with a toroidal bubbly wake
} J.R. Landel, Carlo Cossu, C.P. Caulfield

\section{To cite this version:}

J.R. Landel, Carlo Cossu, C.P. Caulfield. Spherical cap bubbles with a toroidal bubbly wake. Physics of Fluids, 2008, 20 (12), pp.122101. 10.1063/1.3026747 . hal-01022809

\section{HAL Id: hal-01022809 \\ https://hal-polytechnique.archives-ouvertes.fr/hal-01022809}

Submitted on 20 Jul 2014

HAL is a multi-disciplinary open access archive for the deposit and dissemination of scientific research documents, whether they are published or not. The documents may come from teaching and research institutions in France or abroad, or from public or private research centers.
L'archive ouverte pluridisciplinaire HAL, est destinée au dépôt et à la diffusion de documents scientifiques de niveau recherche, publiés ou non, émanant des établissements d'enseignement et de recherche français ou étrangers, des laboratoires publics ou privés. 


\title{
AIP | Physics of Fluids
}

\section{Spherical cap bubbles with a toroidal bubbly wake}

\author{
J. R. Landel, C. Cossu, and C. P. Caulfield
}

Citation: Physics of Fluids (1994-present) 20, 122101 (2008); doi: 10.1063/1.3026747

View online: http://dx.doi.org/10.1063/1.3026747

View Table of Contents: http://scitation.aip.org/content/aip/journal/pof2/20/12?ver=pdfcov

Published by the AIP Publishing

\section{Articles you may be interested in}

Numerical investigation of rising bubble wake and shape variations

Phys. Fluids 21, 122102 (2009); 10.1063/1.3271146

On the turbulent structure in the wake of Taylor bubbles rising in vertical pipes

Phys. Fluids 19, 035108 (2007); 10.1063/1.2711478

Transition from spherical cap to toroidal bubbles

Phys. Fluids 18, 052102 (2006); 10.1063/1.2196451

Wake of a spherical bubble or a solid sphere set fixed in a turbulent environment

Phys. Fluids 18, 048102 (2006); 10.1063/1.2191885

Structure and dynamics of the wake of bubbles and its relevance for bubble interaction

Phys. Fluids 11, 1781 (1999); 10.1063/1.870043

\section{A|P| Journal of}




\title{
Spherical cap bubbles with a toroidal bubbly wake
}

\author{
J. R. Landel, ${ }^{1}$ C. Cossu, ${ }^{2, a)}$ and C. P. Caulfield ${ }^{3, b)}$ \\ ${ }^{1}$ École Polytechnique, F-91128 Palaiseau, France and Department of Applied Mathematics \\ and Theoretical Physics, University of Cambridge, Centre for Mathematical Sciences, Wilberforce Road, \\ Cambridge CB3 OWA, United Kingdom \\ ${ }^{2}$ Laboratoire d'Hydrodynamique (LadHyX), CNRS-École Polytechnique, F-91128 Palaiseau, France \\ ${ }^{3}$ BP Institute and Department of Applied Mathematics and Theoretical Physics, University of Cambridge, \\ Madingley Rise, Madingley Road, Cambridge CB3 OEZ, United Kingdom
}

(Received 9 June 2008; accepted 15 October 2008; published online 2 December 2008)

The prediction of the rise speed of large buoyant bubbles is a fundamental fluid mechanics problem relevant to a number of applications ranging from carbon sequestration technology to chemical engineering or astrophysics. Single large bubbles typically have a spherical cap shape with bubbles of larger volume rising faster than the ones of smaller volume. However, except in well-controlled experiments, the released gas splits into a leading cap bubble, followed by a crown of satellite bubbles that can contain up to $50 \%$ of the total volume of gas. We find that in this case the satellite bubbles rearrange in a characteristic toroidal crown and the leading bubble takes a lenticular shape. The rise speeds of these multipart bubble systems and the ratios of the torus radii to the leading cap curvature radii are quite constant and predictable in the mean and are furthermore independent of the gas partitioning between the leading lenticular bubble and the crown of satellite bubbles. We also find that this multipart bubble system rises slightly faster than a single cap bubble with the same total injected volume of gas. (C) 2008 American Institute of Physics. [DOI: 10.1063/1.3026747]

\section{INTRODUCTION}

Understanding and predicting the dynamics of the rise in large volumes of buoyant gas is important in many applications ranging from radio emissions in astrophysical objects $^{1-3}$ to chemical engineering ${ }^{4-6}$ or carbon sequestration technology. For instance, in problems related to $\mathrm{CO}_{2}$ natural or artificial sequestration in lakes or oceans, a fundamental concern is the possibility of leakage. There is an attendant natural interest in the prediction of the rise speed of a given volume $V$ of gas into water and of the rate of dissolution of that gas into water. If this gas rises at slow speeds, then it can dissolve over a short distance. ${ }^{7-9}$ However, if the rise speed is sufficiently large, then $\mathrm{CO}_{2}$ can contaminate greater depths of water, and indeed it might even reach the surface and be rereleased in the atmosphere. The prediction of the final behavior therefore depends both on the prediction of rise speed of the gas and on the understanding of its shape and its exchange rates.

Single bubbles of large enough volume (typically more than $5 \mathrm{~cm}^{3}$ for air in water ${ }^{10}$ ), for which the effects of surface tension are weak, usually have a spherical cap shape and their behavior is relatively well understood. ${ }^{10-12}$ In particular, the rise speed $U$ is related to the cap bubble frontal curvature radius $R$ by the famous relation of Davies and Taylor, ${ }^{11} U$ $=(2 / 3)(g R)^{1 / 2}$, where $g$ is the gravity acceleration. As explained by Batchelor, ${ }^{10}$ this relationship is derived from an equilibrium condition given by Bernoulli's theorem applied locally at the leading edge of the bubble. For sufficiently large Reynolds numbers, the wake of the bubble is turbulent

\footnotetext{
a)Electronic mail: carlo.cossu@ladhyx.polytechnique.fr.

${ }^{b)}$ Electronic mail: c.p.caulfield@ bpi.cam.ac.uk.
}

and its mean rise speed is steady and is independent of the Reynolds number. ${ }^{12}$

Experience, however, shows that single cap bubbles can be obtained only using extreme care in their generation process. It is known that the release of a spherical volume of still air in water leads to the formation of a leading cap bubble, followed by a toroid of smaller satellite bubbles. ${ }^{13}$ This kind of complex configuration, which is likely to occur in all but the most well-controlled conditions, has not yet been investigated in detail and, for instance, it is not considered in models of $\mathrm{CO}_{2}$ dissolution in the sea. ${ }^{8}$ The scope of the present study is therefore to understand the main characteristics of this most probable complex system formed by the leading cap bubble and its crown of satellite bubbles. Many questions arise about this system. In particular, we wish to determine as to whether or not this configuration is stable; whether its main characteristics (such as the main radius, the distance of the toroid from the leading bubble, and the rise speed of the system) are predictable; and to what extent these characteristics depend on the details of each configuration. To answer these three fundamental questions, we have therefore conducted an experimental analysis. In Sec. II we describe the experimental apparatus and the measurement procedures used to obtain the results reported in Sec. III and discussed in Sec. IV.

\section{EXPERIMENTAL PROCEDURE}

The experimental apparatus, sketched in Fig. 1, is aimed at creating single and crowned cap bubbles by filling an inverted cup with a known volume of air $V_{\text {in }}$ (ranging from 5 to $160 \mathrm{~cm}^{3}$ ). The cup can then be rotated to release the gas. The bubble motion was recorded by a high-speed digital 


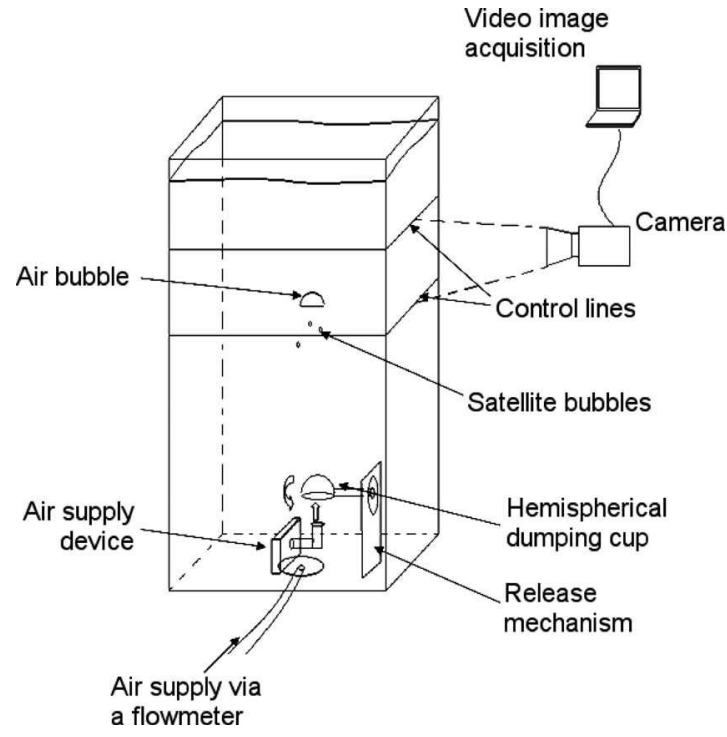

FIG. 1. Experimental apparatus. Air bubbles are generated inside a 75 $\times 75 \times 120 \mathrm{~cm}^{3}$ tank filled with water by injecting a volume $V_{\text {in }}$ of air, measured with a flow meter with $3 \%$ accuracy, into an inverted cup which is then rotated about a horizontal axis to release the gas. Two different cups have been used: the first $(\mathrm{C} 1)$ is a spherical cap cut from a sphere with a curvature radius of $7.5 \mathrm{~cm}$ and rotates about an axis set at $4.5 \mathrm{~cm}$ from its edge, while the second $(\mathrm{C} 2)$ is of half-spherical shape with a curvature radius of $5.5 \mathrm{~cm}$ and rotates on its diameter at $5.5 \mathrm{~cm}$ from its edge. The bubble motion is recorded by a high frame rate (262 frames/s) video camera (Dalsa DA6-512) located at half-tank height.

video camera. Standard single cap bubbles, like the one shown in Fig. 2, were obtained using a large cup (C1), rotated with extreme care. The use of the smaller cup (C2) generally resulted in the formation of cap bubbles crowned with a toroid of satellite bubbles trapped in their wake, as shown in Fig. 3. The dimensions of the bubbles and their rise speed were computed by image analysis. The lengths were preliminarily calibrated using the image of a scale placed in the filled water tank at the position of the bubbles. For each cap bubble the width $d$ of its base and its distance $h$ from the leading stagnation point was measured. The cap radius of curvature and subtended half-angle were then calculated from $R=\left[h^{2}+(d / 2)^{2}\right] / 2 h$ and $\theta_{m}=\arccos (1-h / R)$. The main diameter $2 R_{c}$ of the crown was estimated by measuring the distance of the rightmost and leftmost extreme satellite bubble edges. A measure of the volume $V_{\text {sat }}$ of gas contained

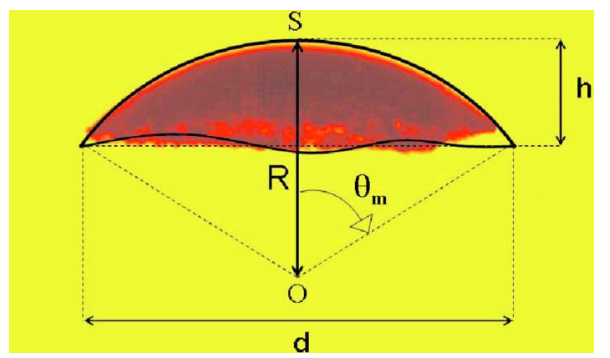

FIG. 2. (Color online) Geometry of a single spherical cap bubble. Picture of a single spherical cap bubble of air $\left(V=68 \mathrm{~cm}^{3}\right)$ rising in still water at constant speed $U . R$ is the radius of curvature of the bubble at the front stagnation point $\mathrm{S}$, while $\theta_{m}$ is its subtended half-angle; $h$ and $d$ are the height and width of the bubble, respectively.

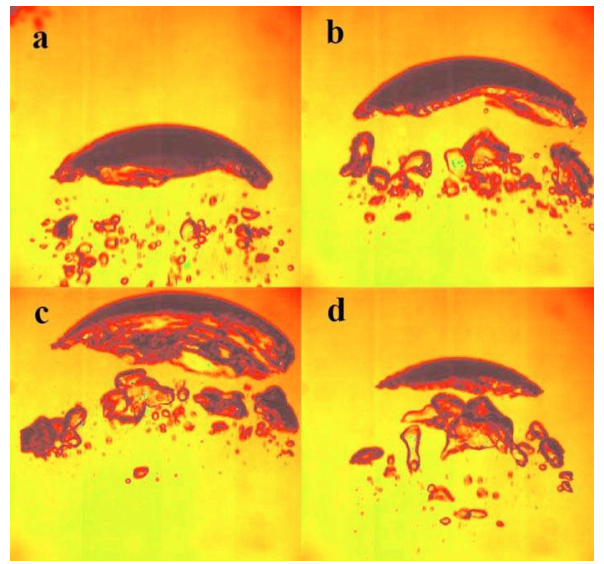

FIG. 3. (Color online) Pictures of typical crowned cap bubbles with, respectively, (a) $V_{\text {in }}=114 \mathrm{~cm}^{3}, V_{\text {sat }} / V_{\text {in }}=0.05$; (b) $V_{\text {in }}=126 \mathrm{~cm}^{3}, V_{\text {sat }} / V_{\text {in }}=0.15$; (c) $V_{\text {in }}=144 \mathrm{~cm}^{3}, V_{\text {sat }} / V_{\text {in }}=0.3$; and (d) $V_{\text {in }}=66 \mathrm{~cm}^{3}, V_{\text {sat }} / V_{\text {in }}=0.5$.

in the crown of the crowned cap bubbles was obtained by associating each satellite bubble appearing on a recorded image with the volume of a sphere of diameter equal to its largest dimension. As explained below, the main properties of the bubbles, such as their Froude number, were found to be insensitive to $V_{\text {sat }} / V_{\text {in }}$ for $V_{\text {sat }} / V_{\text {in }}>0.1$, and therefore we have not looked for more precise measures of $V_{\text {sat }}$. Velocities are computed by selecting the horizontal coordinate where the front stagnation point was located in the initial image for that bubble. The position of the front of the bubble cap at the selected horizontal coordinate was then tracked on the images and it was found to be linear with time, the proportionality factor giving the rise speed $U$.

\section{RESULTS}

\section{A. The multipart "crowned" cap bubble system}

From repeated experiments with the two different cups, it is observed that the ratio $V_{\text {sat }} / V_{\text {in }}$ ranges approximately from 0.01 to 0.5 with an average value of 0.15 without any apparent correlation with $V_{\text {in }}$. Each observed crowned cap bubble system is therefore different from the others, the precise quantitative details of each configuration being strongly dependent, in an apparently nonreproducible way, on the initial formation phase. Indeed, various formation mechanisms were observed, depending on the precise details of the initial conditions. Under certain circumstances, a strong vertical jet developed, which penetrated the upper surface of the initial bubble, disrupting it sufficiently to form a primary spherical cap bubble and a toroidal ring of satellite bubbles. This mechanism has a clear connection to the previously considered formation mechanism ${ }^{13}$ of toroidal bubbles from sufficiently large three-dimensional bubbles. On the other hand, crowned bubble systems were also formed when the jet was not sufficiently strong to penetrate the upper surface of the original bubble. In such circumstances, the crown of satellite bubbles appeared to be formed by the strong toroidal vorticity in the liquid which is shed from the trailing edge of the accelerating initial bubble. Videos showing both these mechanisms are available online as supplemental material. ${ }^{14}$ 
Fundamentally, only when great care was taken to minimize initial external perturbations, and the larger (C1) cup was used so that the initial bubble was as close as possible in geometry (and in particular in its subtended half-angle) to its final form, did we observe single spherical cap bubbles as opposed to the crowned cap bubble system.

However, further analysis reveals that some features, which are common to all the observed crowned bubble systems, are actually reproducible irrespective of the specific details of the initial formation mechanism: (a) although the single satellites in the crown can break up and merge with each other and with the leading cap bubble, they remain trapped in the global system and the total volume of gas of the crowned bubble system remains constant; (b) both the main radius $R_{c}$ of the toroidal crown and its distance $H_{c}$ from the forward stagnation point on the leading cap bubble are steady in the mean and proportional to the curvature radius $R$ of the leading cap bubble with $R_{c} / R=0.835 \pm 7 \%, H_{c} / R$ $=0.82 \pm 7 \%$; (c) the leading cap angles of the crowned bubbles (mean value $\bar{\theta}_{m}=45^{\circ}$ ) are approximately the same as those of single cap bubbles (mean value $\bar{\theta}_{m}=47^{\circ}$ ); and (d) while the lower surface of single cap bubbles is essentially flat, the lower surface of the leading bubble in the crowned bubble system is indented with a second characteristic radius of curvature giving it a lenticular shape. The bubbles of the crowned systems therefore arrange themselves in a configuration which is very specific and has reproducible mean characteristics representing in all respects a well-defined multipart bubble system, as shown in Fig. 3.

\section{B. Rise speed of multipart crowned cap bubble systems}

All the observed bubbles, single or crowned, rise at constant speed $U$ in the measuring window. It is known ${ }^{12,15}$ that for the large injected volumes of gas $\left(5 \mathrm{~cm}^{3}<V_{\text {in }}\right.$ $<160 \mathrm{~cm}^{3}$ ) considered here, the velocity $U$ of a single bubble is not sensitive to variations in surface tension or of molecular viscosity of the fluid and, as single bubbles of given shape have a unique characteristic length $V^{1 / 3}$, dimensional analysis predicts that the Froude number $\mathrm{Fr}$ $=U g^{-1 / 2} V^{-1 / 6}$ is constant. ${ }^{12}$ A theoretical prediction for the Froude number

$$
\mathrm{Fr}^{(\mathrm{SCB})}=\frac{2}{3}\left[\pi f\left(\theta_{m}\right)\right]^{-1 / 6}
$$

is obtained by using the expression for the velocity $U$ $=(2 / 3)(g R)^{1 / 2}$ proposed by Davies and Taylor ${ }^{11}$ and the expression for the volume of a single perfectly spherical cap bubble with flat lower surface $V=\pi R^{3} f\left(\theta_{m}\right)$, where

$$
f\left(\theta_{m}\right)=\frac{1}{3}\left[2-3 \cos \left(\theta_{m}\right)+\cos ^{3}\left(\theta_{m}\right)\right] .
$$

The measured speeds $U$ of the single cap bubbles, plotted versus $V_{\text {in }}$ in Fig. 4, match with this theoretical prediction very well. Indeed, the Froude number that best fits all the single cap bubble data, $\overline{\mathrm{F}} \mathrm{r}=0.8155$, deviates by less than $1 \%$ from the theoretically predicted value $\mathrm{Fr}=0.8223$ obtained using Eq. (1) with the average of the measured cap angles $\bar{\theta}_{m}=47^{\circ}$.

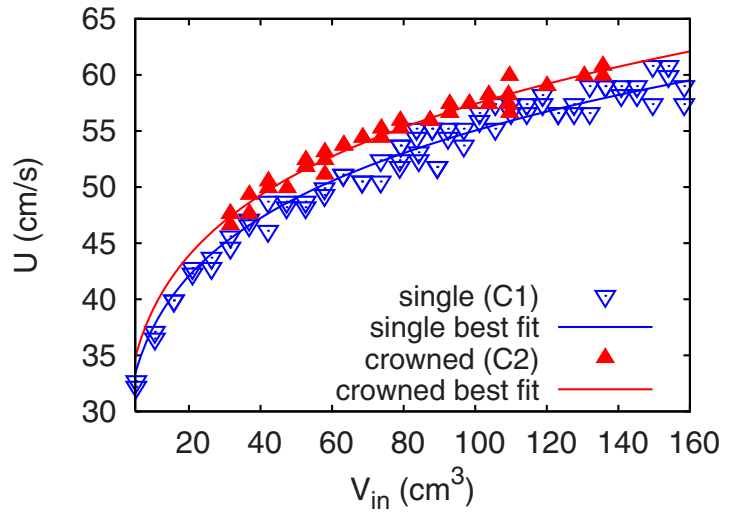

FIG. 4. (Color online) Rise speed $U$ vs total injected volume of air for single cap bubbles $\left(V_{\text {sat }} / V_{\text {in }}<0.05\right)$ generated with the $\mathrm{C} 1$ cup and crowned cap bubbles $\left(V_{\text {sat }} / V_{\text {in }}>0.1\right)$ generated with the $\mathrm{C} 2$ cup. The best-fit curves have been computed by assuming $U=\operatorname{Fr} g^{1 / 2} V_{\text {in }}^{1 / 6}$ and then computing the best-fit Froude number $\overline{\mathrm{F}}$. The data used to generate this figure are available online as supplemental material (Ref. 14).

The velocity data relative to the crowned cap bubbles are best plotted, i.e., with minimized scatter, versus the total volume of injected gas $V_{\text {in }}$, as shown in Fig. 4, and not versus the volume of the leading bubble $V_{\text {in }}-V_{\text {sat }}$. As no systematic correlation seems to exist between $V_{\text {sat }}$ and $V_{\text {in }}$, this result implies that the Froude number of crowned bubbles is not very sensitive to the ratio $V_{\text {sat }} / V_{\text {in }}$. To investigate this implication and to check the possible dependence of the Froude numbers corresponding to each specific crowned bubble on the additional length scales introduced by the satellite bubbles, we have plotted (in Fig. 5) the "individual" Froude numbers $\mathrm{Fr}=U g^{-1 / 2} V_{\text {in }}^{-1 / 6}$ corresponding to each single data point in Fig. 4 versus the corresponding ratio $V_{\text {sat }} / V_{\text {in }}$. Not only is there no clear influence of $V_{\text {sat }} / V_{\text {in }}$ on the individual values of $\mathrm{Fr}$ but also the scatter for crowned bubbles $\left(V_{\text {sat }} / V_{\text {in }}>0.1\right)$ is essentially the same as the scatter for single bubbles $\left(V_{\text {sat }} / V_{\text {in }}<0.05\right)$. The approximately $5 \%$ scatter is consistent with that reported for similar

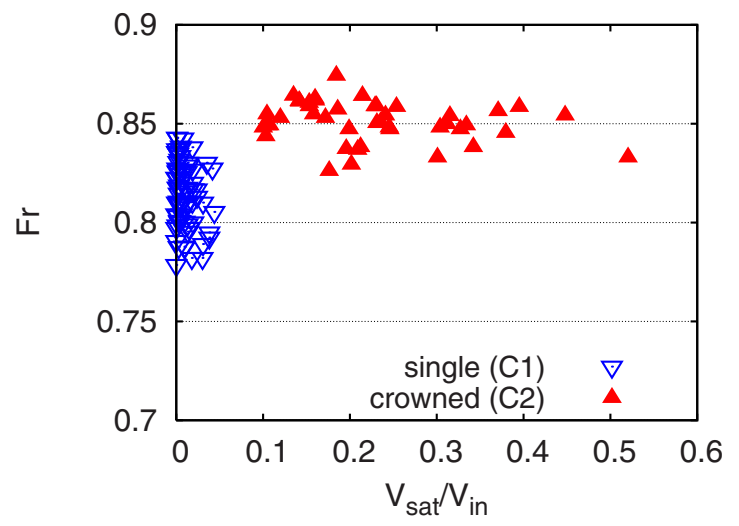

FIG. 5. (Color online) Measured individual Froude numbers of single and crowned cap bubbles plotted vs the ratio of the estimated volume $V_{\text {sat }}$ contained in the satellite bubbles to the total injected volume of gas $V_{\text {in }}$. The data used to generate this figure are available online as supplemental material (Ref. 14). 
experiments. $^{11,12,15}$ These results underline again that crowned bubbles must be considered as well-defined and reproducible multipart bubble systems.

\section{DISCUSSION AND CONCLUSIONS}

For a given total volume of injected gas $V_{\text {in }}$, a single cap bubble is predicted to have speed $U^{(\mathrm{SCB})}=\mathrm{Fr}^{(\mathrm{SCB})} g^{1 / 2} V_{\text {in }}^{1 / 6}$ (where $\mathrm{Fr}^{(\mathrm{SCB})}=0.8223$ for $\theta_{m}=47^{\circ}$ ). The use of the same formula to predict the speed of the leading bubble (of volume $\left.V_{\text {in }}-V_{\text {sat }}\right)$ of a crowned system would predict $U$ $=\mathrm{Fr}^{(\mathrm{SCB})} g^{1 / 2}\left(V_{\text {in }}-V_{\text {sat }}\right)^{1 / 6}$ a rise speed lower than that of a single cap bubble of the same total volume of injected gas $V_{\text {in }}$. However, from Figs. 4 and 5 the opposite is observed: Crowned cap bubble systems not only do not rise slower but they even rise slightly faster than single cap bubbles. The best-fit Froude number to the crowned bubble data is $\overline{\mathrm{Fr}}$ $=0.8508$, corresponding to a velocity greater by slightly more than $4 \%$ in the mean than the velocity of a single cap bubble of the same total volume of injected gas. This apparent paradox can be solved by remarking that the formula used for single cap bubbles assumes a spherical cap shape with a flat lower surface, while the leading cap bubble of the crowned system has a concave rear part leading to an apparently drag-reducing lenticular shape. It is thus important to investigate the mechanism by which the lenticular shape develops, and indeed whether it is the lenticular shape that is the primary cause of the apparent drag reduction.

All the observed bubbles (single and crowned) have a steady mean speed that is selected by the equilibrium of the buoyancy forces associated with the bubbles and their drag, mainly associated with turbulent dissipation in the liquid wake. $^{10,12}$ Since the crown of satellite bubbles rises at the same speed as the leading bubble, the mean buoyancy force $\rho g V$ is the same for the single cap and the crowned cap bubble systems of the same global gas volume $V$. As the total buoyancy force is the same in both cases, the slight increase in $U$ in the presence of satellite bubbles can therefore only be explained by a modification in the wake flow resulting in a slight decrease in turbulent dissipation, and hence of the mean drag. Furthermore, this decrease in the turbulent drag forces can be uniquely attributed to the presence of the bubbles in the wake because no increase in $U$ is observed in the absence of satellite bubbles. In addition, the frontal radius of curvature of single and crowned cap bubble systems is related to the mean rise speed by the formula of Davies and Taylor, ${ }^{11} U=(2 / 3)(g R)^{1 / 2}$. This relationship is well satisfied in our experiments for both single and crowded cap bubbles, as shown in Fig. 6 where the experimentally measured ratio $U /(g R)^{1 / 2}$ is very close to two-thirds and does not depend on $V_{\text {sat }} / V_{\text {in }}$.

In other words, for a given injected volume of air $V_{\text {in }}$, in the presence of satellite bubbles, the frontal radius of curvature of the leading cap bubble is slightly larger (by approximately $10 \%$ ) because of the slight increase in the bubble velocity $U$. As shown in Fig. 7, $\theta_{m}$ does not change significantly (from $47^{\circ}$ for the single spherical cap bubbles to $45^{\circ}$ for the crowned cap bubble systems). The volume of the leading bubble is smaller than the volume of a single cap

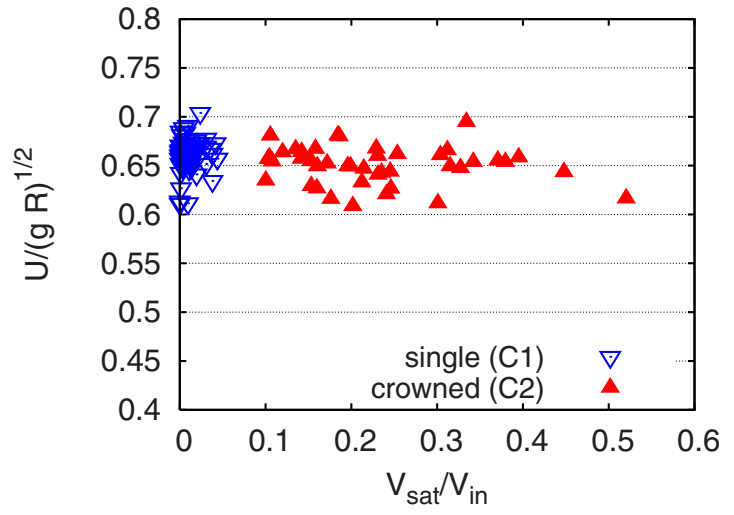

FIG. 6. (Color online) Measured individual Davies and Taylor ratios $U / \sqrt{g R}$ of single and crowned cap bubbles plotted vs the ratio of the estimated volume $V_{\text {sat }}$ contained in the satellite bubbles to the total injected volume of gas $V_{\text {in }}$. The ratio predicted by the theory of Davies and Taylor (Ref. 11) is $2 / 3=0.666 \overline{6}$. The data used to generate this figure are available online as supplemental material (Ref. 14).

bubble with the same total $V_{\text {in }}$, the difference in gas volume being associated with the satellite bubbles. The only way to satisfy all these conditions (i.e., a greater radius of curvature, an unchanged subtended angle, and a smaller volume) is for the leading bubble to have a nonflat lower surface, thus explaining the observed lenticular shape. Therefore, it appears reasonable to think of the observed lenticular shape as a consequence rather than the cause of the implied reduced drag of the crowned cap bubble system.

The exact mechanism by which the drag is slightly reduced in the presence of the satellite bubbles is presently unknown and only conjectures can be made by noting some similarities with other findings. A first idea could be to relate this situation to the large rise speeds observed for swarms of bubbles of large void fraction rising in tubes. ${ }^{16}$ However, this situation is very different from the one considered here. All the bubbles have comparable volumes in the tube flow, and are much smaller than the ones we observe. Also, all the bubbles in the tube are at the same time "leaders" and "followers." Although numerical studies of two-bubble interactions ${ }^{5,6}$ clearly show an acceleration effect on follower bubbles, that could only explain why the satellites are cap-

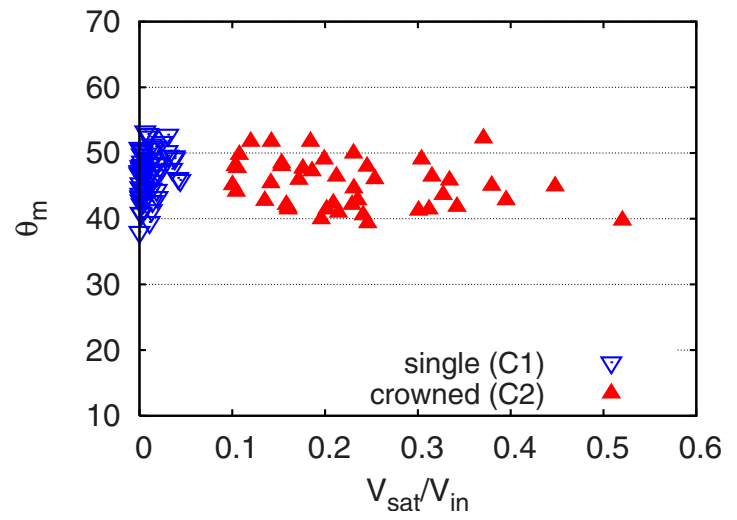

FIG. 7. (Color online) Measured opening angles $\theta_{m}$ of single and crowned cap bubbles plotted vs the ratio $V_{\text {sat }} / V_{\text {in }}$. The data used to generate this figure are available online as supplemental material (Ref. 14). 
tured in the wake of the leading cap bubble. However, there is no explicit observation of an acceleration of the leader bubble. A second observation that can be related to our findings is the drag reduction observed in turbulent wallbounded shear flows in the presence of small bubbles. ${ }^{17,18}$ Although in the present study there are no rigid walls, numerical simulations ${ }^{19}$ indicate that in the case of homogeneous turbulence in the presence of microbubbles the turbulent dissipation can be reduced up to $4 \%$ and that even larger reductions can be expected for larger bubbles. ${ }^{20}$ It is actually not clear in the case of crowned cap bubble systems whether the reduction in the drag forces directly results from the same bulk-type mechanism or if it occurs indirectly by a slight modification of the wake geometry. The complete understanding of the complex flow in the wake is indeed a challenge, so future work could involve wake visualization to investigate further the drag reduction effect observed in crowned cap bubble systems.

\section{ACKNOWLEDGMENTS}

J.R.L. thanks the Department of Applied Mathematics and Theoretical Physics (DAMTP), University of Cambridge for its hospitality. C.C. acknowledges partial support from the French Ministry of Foreign Affairs and Churchill College (University of Cambridge) for its hospitality during his stay in Cambridge. Thanks are due to Andrew Pluck of the BP Institute and David Page-Croft of the G. K. Batchelor (GKB) Laboratory (DAMTP) for experimental support. The technical assistance and advice of Stuart Dalziel, Director of the GKB Laboratory, are also gratefully acknowledged.

${ }^{1}$ S. Gull and K. Northover, "Bubble model of extragalactic radio sources," Nature (London) 244, 80 (1973).

${ }^{2}$ E. Churazov, M. Brüggen, C. Kaiser, H. Böhringer, and W. Forman, "Evolution of buoyant bubbles in M87," Astrophys. J. 554, 261 (2001).

${ }^{3}$ A. Fabian, J. Sanders, C. Crawford, C. Conselice, J. Gallagher, and R. Wyse, "The relationship between the optical $\mathrm{H} \alpha$ filaments and the x-ray emission in the core of the Perseus cluster," Mon. Not. R. Astron. Soc. 344, L48 (2003).

${ }^{4}$ J. Davidson, D. Harrison, and J. Carvalho, "On the liquidlike behavior of fluidized beds," Annu. Rev. Fluid Mech. 9, 55 (1977).

${ }^{5}$ R. Krishna, M. Urseanu, J. van Baten, and J. Ellenberger, "Rise velocity of a swarm of large gas bubbles in liquids," Chem. Eng. Sci. 54, 171 (1999).

${ }^{6}$ R. Krishna and J. van Baten, "Simulating the motion of gas bubbles in a liquid," Nature (London) 398, 208 (1999).

${ }^{7}$ P. Brewer, E. Peltzer, G. Friederich, and G. Rehder, "Experimental determination of the fate of rising $\mathrm{CO}_{2}$ droplets in seawater," Environ. Sci. Technol. 36, 5441 (2002).

${ }^{8}$ R. Gangstø, P. Haugan, and G. Alendal, "Parameterization of drag and dissolution of rising $\mathrm{CO}_{2}$ drops in seawater," Geophys. Res. Lett. 32, L10612, DOI:10.1029/2005GL022637 (2005).

${ }^{9}$ P. Brewer, B. Chen, R. Warzinki, A. Baggeroer, E. Peltzer, R. Dunk, and P. Walz, "Three-dimensional acoustic monitoring and modeling of a deepsea $\mathrm{CO}_{2}$ droplet cloud," Geophys. Res. Lett. 33, L23607, DOI:10.1029/ 2006GL027181 (2006).

${ }^{10}$ G. K. Batchelor, An Introduction to Fluid Dynamics (Cambridge University Press, Cambridge, UK, 1967).

${ }^{11}$ R. Davies and G. Taylor, "The mechanics of large bubbles rising through extended liquids and through liquids in tubes," Proc. R. Soc. London, Ser. A 200, 375 (1950).

${ }^{12}$ P. Wegener and J. Parlange, "Spherical-cap bubbles," Annu. Rev. Fluid Mech. 5, 79 (1973).

${ }^{13}$ J. Walters and J. Davidson, "The initial motion of a gas bubble formed in an inviscid liquid Part 2: the three-dimensional bubble and the toroidal bubble," J. Fluid Mech. 17, 321 (1963).

${ }^{14}$ See EPAPS Document No. E-PHFLE6-20-028811. There is a README.txt file, two videos showing different formation mechanisms for crowned cap bubble systems, and two data files containing the data used to generate Figs. 4-7. For more information on EPAPS, see http:// www.aip.org/pubservs/epaps.html.

${ }^{15} \mathrm{D}$. Bhaga and M. Weber, "Bubbles in viscous liquids: shapes, wakes, and velocities," J. Fluid Mech. 105, 61 (1981).

${ }^{16}$ M. Simonnet, C. Gentric, E. Olmos, and N. Midoux, "Experimental determination of the drag coefficient in a swarm of bubbles," Chem. Eng. Sci. 62, 858 (2007).

${ }^{17}$ N. Madavan, S. Deutsch, and C. Merkle, "Reduction of turbulent skin friction by microbubbles," Phys. Fluids 27, 356 (1984).

${ }^{18}$ T. van den Berg, S. Luther, D. Lathrop, and D. Lohse, "Drag reduction in bubbly Taylor-Couette turbulence,” Phys. Rev. Lett. 94, 044501 (2005).

${ }^{19}$ I. Mazzitelli, D. Lohse, and F. Toschi, "The effect of microbubbles on developed turbulence," Phys. Fluids 15, L5 (2003).

${ }^{20}$ T. Lo, V. L'vov, and I. Procaccia, "Drag reduction by compressible bubbles," Phys. Rev. E 73, 036308 (2006). 